\title{
First Principle Study on the Electronic Structure of FeSe Under Pressure
}

\author{
M. A. Afrassa Habtamu Beyene \\ Adama Science and Technology University
}

\begin{abstract}
In Physics, one of the current hot research related to material for energy storage problem is finding superconducting materials that can work at room temperature. In this work we study the properties of FeSe superconductor under pressure. We have performed density functional theory(DFT) calculations as implemented in QUANTUM ESPRESSO. All calculation were executed using the tetragonal leadoxide or P4/nmm structure, and we used experimental value of lattice parameters a and $\mathrm{c}$. We found that superconducting transition temperature (Tc) of FeSe increases sensitively under pressure. We concluded that the change in lattice parameter and the increment in DOS can be an evidence for high Tc . Furthermore, we compared the result of ferro magnetic and anti feromgnetic ordering and we found that the Fermi energy, total energy and the Tc is higher for anti ferromagnetic ordering calculations. The result shows that the lowest occupied state is in ant ferromagnetic order, in agreement with already published.
\end{abstract}

Keywords: Superconductivity (SC), Band structure, Pressure and Transition Temperature (Tc).

DOI: $10.7176 /$ APTA/77-04

Publication date:May $31^{\text {st }} 2019$

\section{Introduction}

Superconductivity has been observed in different class of materials since the first report in 1911. The phenomena has been studied theoretically and 1experimentally. The reports showed that the superconducting critical temperature Tc raised under the actions of doping, and pressure. In 2008 a new superconductor( $\left(\mathrm{LaF}_{\mathrm{e}} \mathrm{AsO} \mathrm{1-x}_{\mathrm{x}} \mathrm{F}_{\mathrm{x}}\right)$ was found that contains magnetic element, Iron[1]. This was one of the important discovery in the history of Superconductivity. After this report the study of superconductivity shifted its focus from ceramic material to ironbased material.

One of the simplest crystal structure in iron based superconductors (IBS) is the so called "11" family, example, FeSe, as reviewed in ref.[2]. In these materials the mechanism is not yet clearly understood but it is believed that it has magnetic origin $[3,4,5,6]$. Understanding the magnetic nature and its relation with the onset of superconductivity has been a key question. From the experimental point of view FeSe system is preferable due to the fact that it does not contain the toxic element, Arsenic and the possibility of getting high-quality single crystals. It was reported that Tc with various techniques reaches $8 \mathrm{k}-70 \mathrm{k}$ [7].

It has been reported that superconductivity in FeSe induced under pressure [7,8]. Based on the reports it is clearly indicated that the value of Tc is strongly dependent on the applied pressure. But the effect of pressure on the lattice parameter is not well reported. It is well known that the electronic structure of such systems can provide insight into the superconducting properties of materials[9]. In this work we applied Density Functional theory(DFT) as implemented in Quantum espresso. This technique leads to obtain electronic band structure, Density of state(DOS), and Fermi surfaces[10-13]. Density of State(DOS) in superconductivity is an important parameter to understand several properties. If the number of DOS near the Fermi level is changing, for instance by doping, then the properties of the material may change dramatically. Lattice parameters are highly linked with electronic structural properties of super- conductors. FeSe can be considered as reference materials to study effect of pressure(in this case lattice parameter change) and other superconducting properties of other Fe-based superconductors.

In general our research addressed to improving the basic understanding of the properties of FeSe under pressure through electronic structural calculations.

\section{Computational details}

In this research we applied the first principle method in density functional theory(DFT)as implemented in quantum espresso. We selected the suitable pseudo potentials for Fe, and Se from alternative pseudopod-potential library in order to obtain high quality and meaningful results on the behavior of selected correlated-electron compounds. The energy convergence is checked with respect to the cutoff energies and k-point sampling. The initial tetragonal lattice parameters of FeSe in our calculations are based on the experimental value under ambient conditions: $a=b$ $=3.765[\mathrm{a} . \mathrm{u}], \mathrm{c}=5.518[\mathrm{a} . \mathrm{u}][14]$. The bands are plotted along the high symmetry direction in the Conventional Tetragonal-TET Brillouin zone, the lattice following the path $\Gamma-\mathrm{X}-\mathrm{M}-\Gamma-\mathrm{Z}-\mathrm{R}-\mathrm{A}-\mathrm{Z}$ [15]. We used 121 k-points mesh in the Brillouin zone. The DOS calculation were performed using tetrahedron method. The 
calculations were done for non magnetic, Ferromagnetic ordering and anti ferromagnetic ordering.

\section{Result and Discussion}

\subsection{Effects of Pressure on lattice parameters}

In this work we present the electronic structure of FeSe under pressure. The geometry optimization was performed for the tetragonal P4/nmm structure of FeSe within the pseudo-potential approach and quantum ESPRESSO program. We calculated the pressure dependences of the crystal lattice parameters. We did for SCF and optimization. These results are in a qualitative agreement with experimental data of Refs.[16-19].

The pressure dependence of structural parameters were summarized in Figures.1-2. With increasing pressure, the lattice parameters a and c,the ratio of cell parameter $\mathrm{c} / \mathrm{a}$, volume of the material $\mathrm{V}$ and Fe-Se distance decreased monotonously

The result shows that the length of a-axis decrease more slowly compared to c-axis. c-axis seen to decrease more at $\mathrm{P} \approx 2 \mathrm{Kbar}$. The $\mathrm{c} / \mathrm{a}$-ratio is plotted as a function of pressure. We observed that the ratio of cell parameter $\mathrm{c} / \mathrm{a}$ decreased as pressure increased. That is the ratio decreases down to 1.4877 at $178 \mathrm{k}$ bar.

As we know the change in lattice parameter result in changing the volume unless we fixed the volume. Here we found that as lattices parameters are decreased the volume is also decreases, while the transition temperature increased with increasing the pressure. This is a good indication how pressure sensitively correlated with the arrangement of crystal structure. This in turn strongly affect the superconducting properties of the Fe-based superconductor particularly FeSe. Fig. 2(b) shows the unit-cell volume of FeSe plotted as function of pressure. Tables 1 shows the calculated value of Fermi energy, total energies and volume of FeSe with vary lattice parameters a and $\mathrm{c}$ under pressure $(\mathrm{P}=0$ to $2 \mathrm{Kbar})$. From this result, we observed the value of volume and Fermi energy increased while total energies decreased by small amount with increasing pressure. The Fermi energy has been calculated for FeSe, as lattice parameters decrease, the Fermi energy also decreased [tables 1 and 2] show the correlation between Fermi energy, lattice parameter total energies and volume with pres sure.
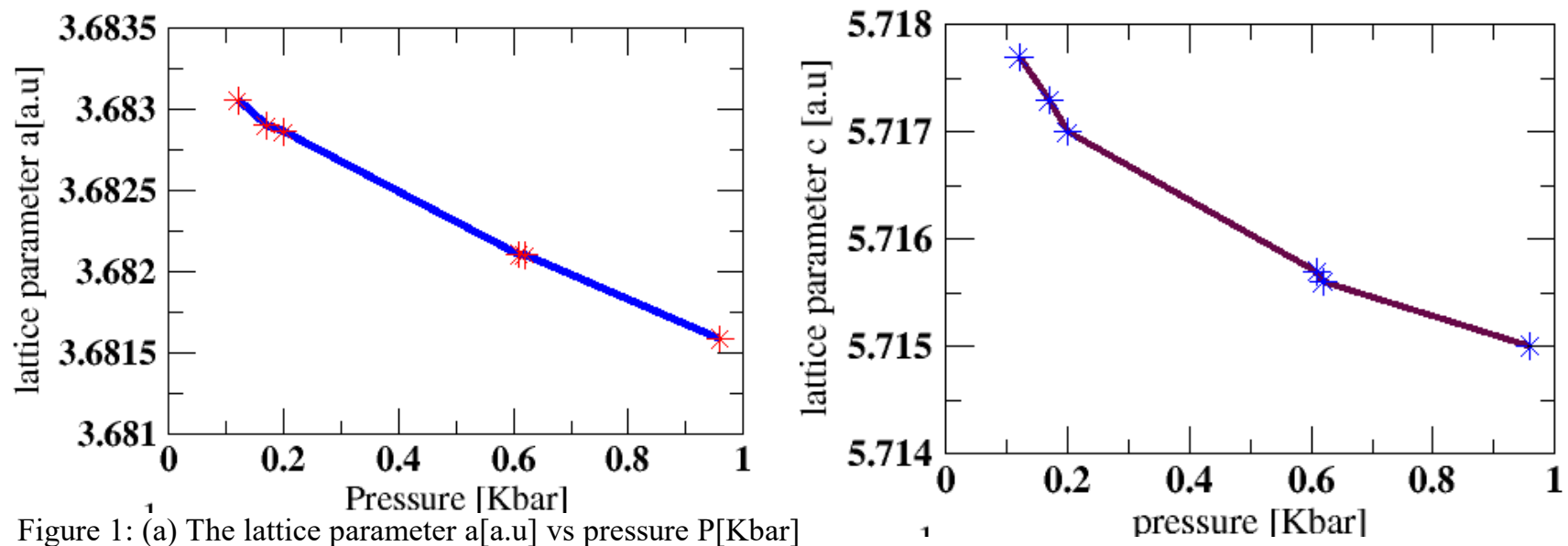

Figure 1: (a) The lattice parameter a[a.u] vs pressure $\mathrm{P}[\mathrm{Kbar}]$ and (b) the lattice parameter c[a.u] vs pressure $\mathrm{P}[\mathrm{Kbar}]$ for FeSe in VC-relax.
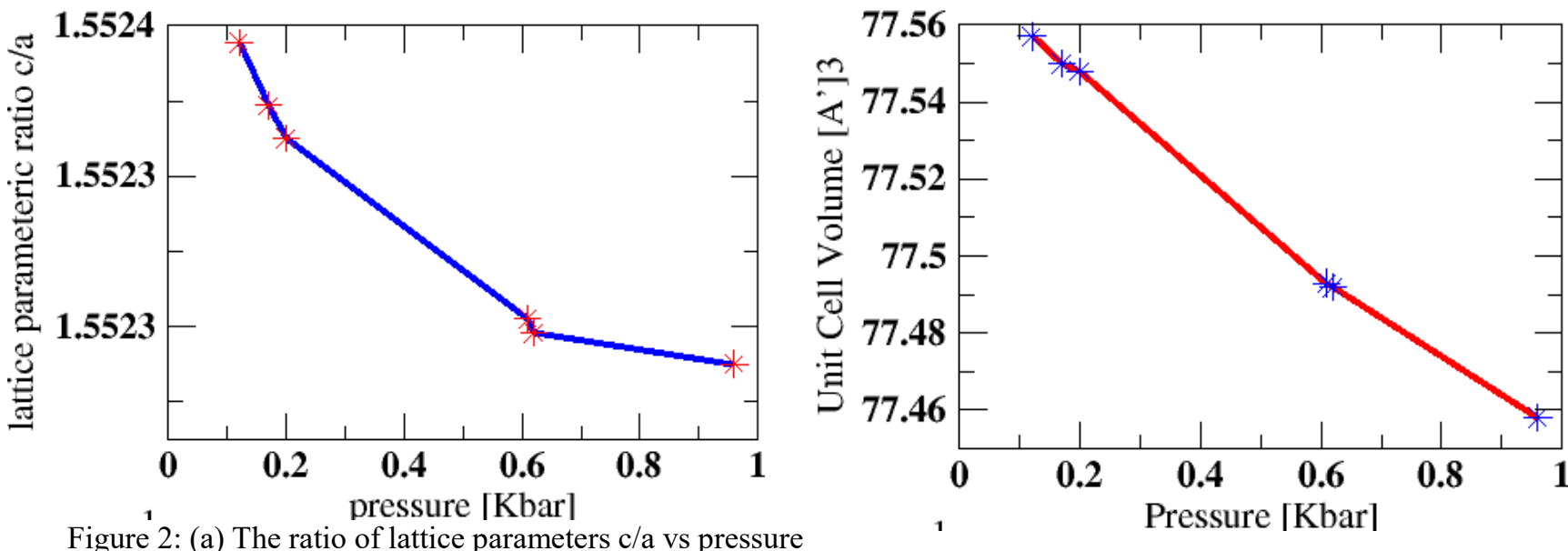

Figure 2: (a) The ratio of lattice parameters c/a vs pressure $\mathrm{P}[\mathrm{Kbar}]$ and (b) unit cell-volume V [ Â 3 ] vs pressure P[Kbar] for FeSe in VC-relax.

The Fermi energy has been calculated for FeSe, as lattice parameters decrease, the Fermi energy also decreased [tables 1 and 2] show the correlation between Fermi energy,lattice parameter total energies and volume 
with pressure.

\section{2. Effect of pressure on Band Structure and DOS of FeSe}

We reproduced the known band structure for FeSe as shown in Fig.3. The Figure shows that the band structure calculated in the space group $\mathrm{P} 4 / \mathrm{nmm}$ with non-spin polarized orbitals in optimization. Most of the features the band structure are similar in both, self consistent field and optimization structure. The Fermi energy is $9.340 \mathrm{eV}$ in self consistent field that would be compared with $8.986 \mathrm{eV}$ for the optimization one at minimum pressure. How ever, at high pressure $\mathrm{p} \approx 2 \mathrm{Kbar}, \mathrm{E}_{\mathrm{F}}$ become $9.37 \mathrm{eV}$ and $10.32 \mathrm{ev}$ respectively.

We also calculated the density of state as shown in Fig 4. The DOS at the Fermi level are slightly enhanced under pressure. Zero pressure values of DOS at $\mathrm{E}_{\mathrm{F}}$ are 2.69(state/cell)eV while at pressures corresponding to the maximum critical temperature, the density's equal to 3.54(state/cell/ev) ( $\mathrm{p} \approx 2 \mathrm{Kbar}$ ).

Table 1: With vary atomic position of FeSe, the lattice parameters(a and c), volume, Fermi energy, total energies and pressure are calculated from optimization.

\begin{tabular}{cccccc}
\hline a[a.u] & $\mathbf{c}[\mathbf{a . u}]$ & $\mathbf{v}\left[\hat{\mathbf{A}}^{3}\right]$ & $\mathbf{E F}[\mathbf{e v}]$ & $\mathbf{E}_{\text {total }}[\mathbf{R y}]$ & $\mathbf{P}[$ Kbar] \\
3.676833615 & 5.4757803884 & 74.028 & 10.3256 & -564.18236501 & 1.94 \\
3.681566350 & 5.7147997000 & 77.458 & 9.0038 & -564.18789490 & 0.96 \\
3.682103727 & 5.7156395467 & 77.492 & 8.9970 & -564.18790545 & 0.62 \\
3.682106347 & 5.7157051703 & 77.493 & 8.9975 & -564.18790470 & 0.61 \\
3.683023793 & 5.7172302634 & 77.552 & 8.9859 & -564.18793605 & 0.20 \\
3.683033850 & 5.7173974160 & 77.555 & 8.9860 & -564.18793604 & 0.17 \\
3.683056993 & 5.7174730082 & 77.557 & 8.9860 & -564.18793604 & 0.12 \\
\hline
\end{tabular}

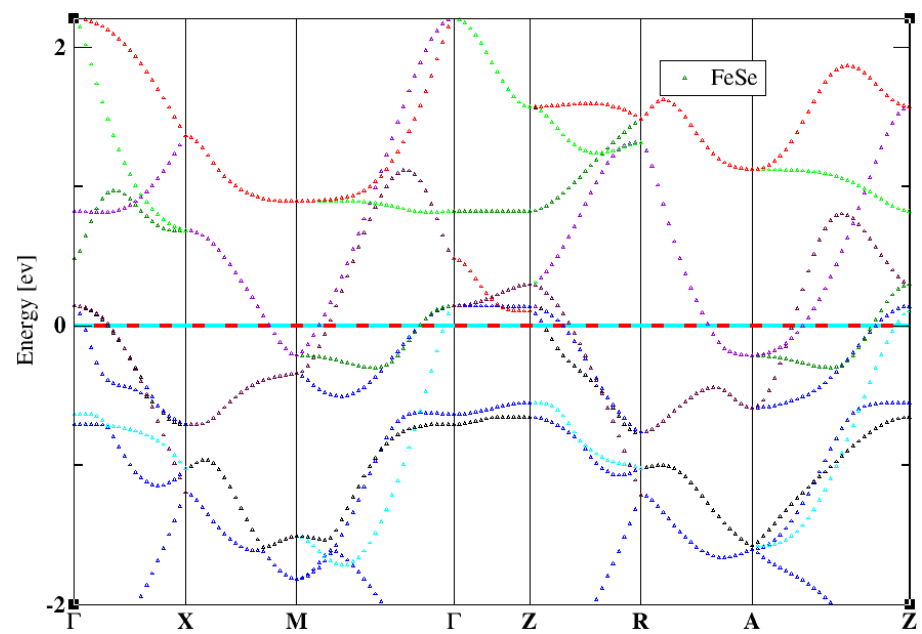

Figure 3: The electronic band structures of FeSe along high- symmetry lines at $\mathrm{p}=1.94 \mathrm{Kbar}$ for optimization.

We calculated the critical temperature Tc based on the well known McMillan equation[20]. In fact we used Debye temperature $\left(\Theta_{\mathrm{D}}=210 \mathrm{~K}\right)$ as suggested in different references. The calculated value of critical temperature , $\mathrm{Tc}=16.75 \mathrm{~K}$ at pressure $\mathrm{P}^{\prime} 2 \mathrm{Kbar}$. The calculated result shows the pressure dependence of Tc . It is found increasing. This value is in good agreement with both theoretical and experimental studied under pressure.

\subsection{Calculation with Magnetic ordering}

It has been reported that Superconductivity in IBS connected with magnetic property. In this work we present a spin polarized calculation for collinear magnetic ordering. As shown in the figure(Fig 5) we present band structure for Anti-ferromagnetic ordering. The band structures are similar with most pnictides that shows a multi band nature. We compared the Fermi energy and the total energy for ferromagnetic and antiferromagnetic ordering calculations. 


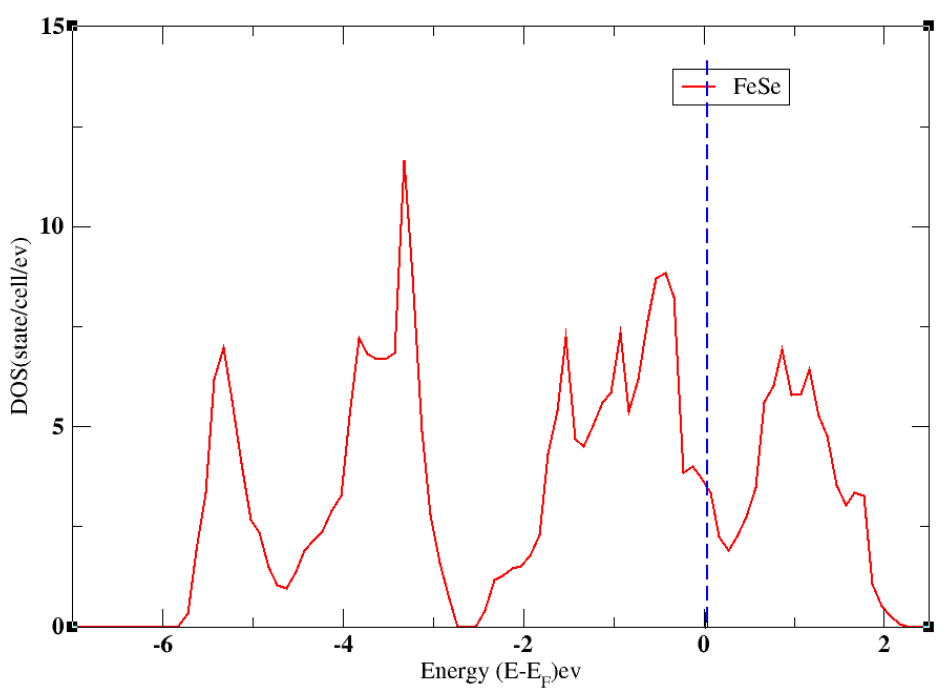

Figure 4: Density of electronic states $\mathrm{N}(\mathrm{E})$ of FeSe at $\mathrm{P}=1.94 \mathrm{Kbar}$. The Fermi level $(\mathrm{E}=0)$ is marked by the vertical broken line.

Table 2: Comparison of Fermi energy and Total energy for Ferro magnetic and anti ferro magnetic ordering.

\begin{tabular}{|c|c|c|}
\hline & Ferro magnetic & Anti ferro magnetic \\
\hline EF & 8.7504 & 8.8418 \\
ET & -563.55141862 Ry & $-563.57067668 \mathrm{Ry}$ \\
DOS & 0.3256 & 1.03081 \\
\hline
\end{tabular}

The energy difference in $\mathrm{eV}$ is $0.262 \mathrm{eV}=262 \mathrm{meV}$. The code calculated the lowest state and the total energy for antiferomagnetic ordering is higher. Based on the value of the DOS(table 2) and using well known MackMillan equation we can predict that $\mathrm{Tc}$ is higher for anti ferromagnetic ordering.

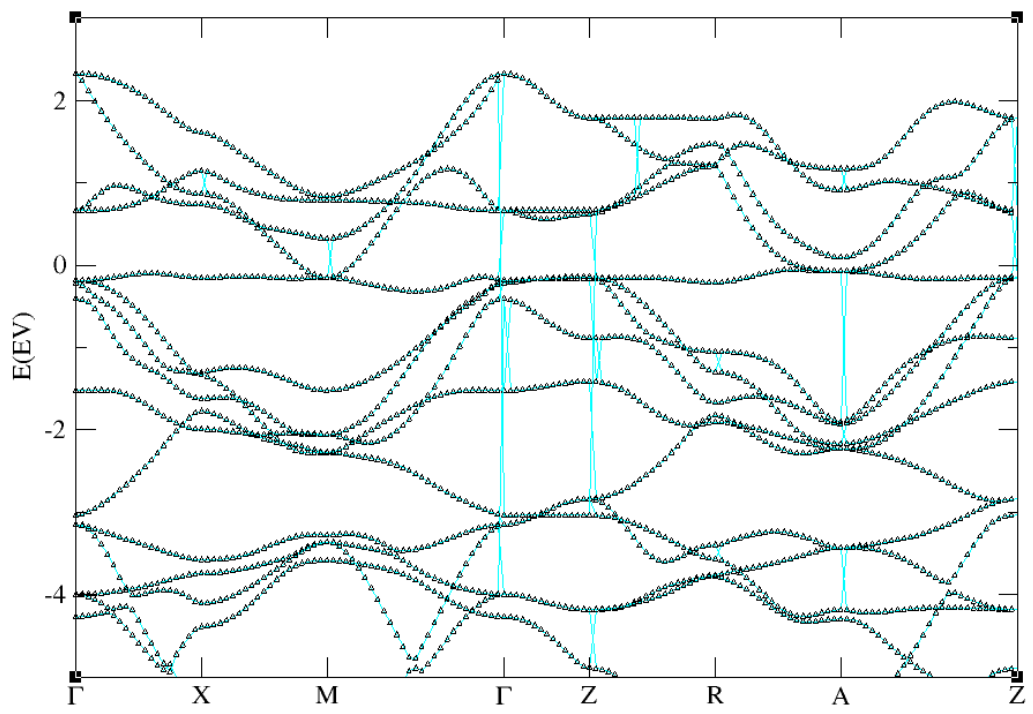

Figure 5: Band structure for FeSe in AFM ordering.

\section{Conclusions}

Applying DFT as implemented in quantum espresso, we make a simulation to obtain a band structure and DOS of FeSe. We have studied the effect of external pressure on both crystal and electronic structures of FeSe superconductors. The effect of pressure on the lattice parameters a and $\mathrm{c}$ are shown in Figures. 1-2. The c-axis is found to be the more shortened and the shortness of this axis is furthermore seen to decrease at $\approx 2 \mathrm{Kbar}$. In contrast, the length of the a-axis decreases smoothly as the pressure increases. The c/aratio is plotted as a function of pressure. The Correlations between pressure and lattice parameters is vary sensitive and the effect is seen on the change in volume and Fermi energy. So, as pressure increase volume would be decreased while the value of T C raised. From our calculation, we seen that a higher DOS at the Fermi level is observed under pressure, The density's at the 
Fermi energy are slightly enhanced under pressure. Zero pressure values of DOS at $E_{F}$ is 2.69(state/cell)(state/cell/ev) while at pressures corresponding to the maximum critical temperature, the density's equal to 3.54(state/cell/ eV) ( $\mathrm{p} \sim=2 \mathrm{Kbar}$ ), which usually improves superconducting properties. We found that the Fermi energy, DOS, and total energy is higher for Antiferromagnetic (AF) ordering. This result of Ferromagnetic and AF spine polarized calculation indicated that FeSe exists in AF ordering at the ground state, in agreement with already published report, e.g. ref. [3].

\section{References}

1] Yoichi Kamihara, Takumi Watanabe, Masahiro Hirano, and Hideo Hosono, $0.5 \mathrm{~cm}$ J. Am. Chem. Soc. 130, 3296 (2008).

[2] G. R. Stewart, Review of Modern Physics 83, (2011).

[3] Fengjie Ma, Wei Ji, Jiangping Hu, Zhong-Yi Lu, and Tao Xiang, 0.5cm PRL 102, 177003 (2009)

[4] I. I. Mazin, D. J. Singh, M. D. Johannes, and M. H. Du., Phys. Rev. Lett. 101, 057003 (2008).

[5] Kazuhiko Kuroki, Seiichiro Onari, Ryotaro Arita, Hidetomo Usui, Yukio Tanaka, Hiroshi Kontan, ibid.101, 087004 (2008)

[6] J. Zhang et al., Phys. Rev. B 79, 220502 (2009).

[7] S. S. N. Rebec, T. Jia, C. Zhang, M. Hashimto, D. H. Lu, R. G. Moore, and Z.X, PRL 118, 067002 (2017).

[8] Brajesh Tiwari, Rajveer Jha, and V. P. S. Awana, AIP ADVANCES 4, 067139 (2014)

[9] T. Mousavi et.al., a review, Materials Science and Technology,30:15, 1929-1943(2014)

[10] A. N. Yaresko, G.-Q. Liu, V. N. Antonov, and O. K. Andersen, Phys. Rev. 79, 144421 (2009).

[11] I.I.Mazine, J. Schkalian, Physica C 469, 614-627 (2009).

[12] D. J. Singh and M.-H. Du, D, Phys.Rev. Lett. 100, 237003 (2008).

[13] M. A. Afrassa and P.Singh, Journal of Advances in Physics, 12, 2016.

[14] Hsu FC, Luo JY, Yeh KW, Chen TK, Huang TW, Wu PM, Lee YC, Huang YL, Chu YY, Yan, Proc. Natl. Acad. Sci. 105, 14262 (2008).

[15] Takahashi, Hiroki, Igawa, Kazumi, Arii, Kazunobu, Kamihara, Yoichi, Hirano, Nature 453, 376-378 (2008).

[16] G.E.Grechnev et al.,SSN 2071-0186. Ukr. J. Phys. Vol.59, No.3 (2014).

[17] Drozdov A.P., Eremets M.I., et al. Nature, 525, P.73-76(2015)

[18] Jasmine N.Millican, DanielPhelan, Evan L.Thomas, Juscelino B.Leao, Elisabeth, Carpenter, Solid State Commun. 149, 707 (2009).

[19] R. S. Kumar, Y. Zhang, S. Sinogeikin, Y. Xiao, S. Kumar,. P. Chow, A. L. Cornelius, J. Phys. Chem. B 114, 12597 (2010).

[20] G. M. Eliashberg, Soviet Phys.-JEPT 11, 696 (1960); 12, 1000 (1961). 